Supplement of Biogeosciences, 16, 3929-3939, 2019

https://doi.org/10.5194/bg-16-3929-2019-supplement

(C) Author(s) 2019. This work is distributed under

the Creative Commons Attribution 4.0 License.

(c) (1)

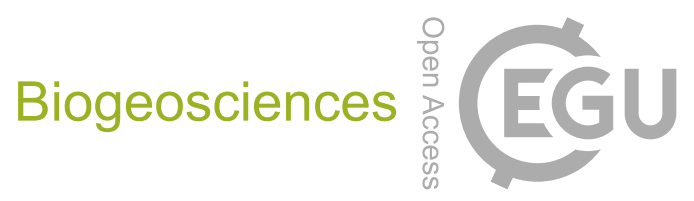

Supplement of

\title{
Spatial changes in soil stable isotopic composition in response to carrion decomposition
}

Sarah W. Keenan et al.

Correspondence to: Sarah W. Keenan (sarah.keenan@sdsmt.edu) and Jennifer M. DeBruyn (jdebruyn@utk.edu)

The copyright of individual parts of the supplement might differ from the CC BY 4.0 License. 


\section{Supporting Information}

(a)

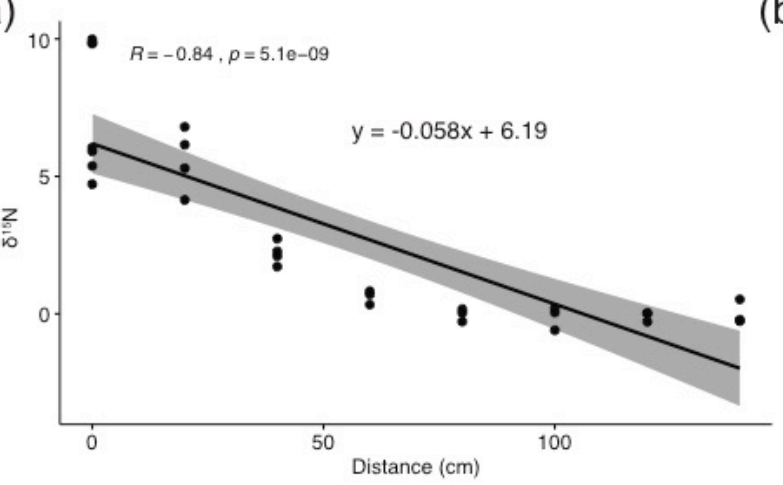

(b)

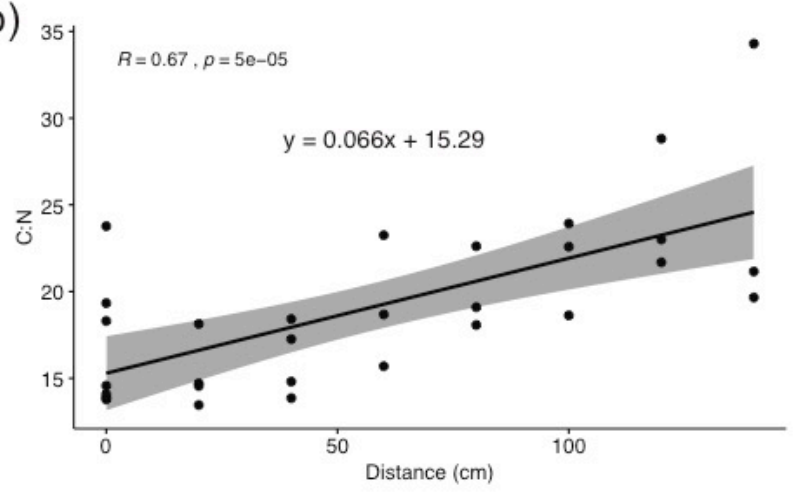

Figure S1: Linear correlation analysis of $\delta^{15} \mathrm{~N}$ and C:N as a function of distance from the hotspot center $(0 \mathrm{~cm})$. (a) There is a roughly linear decrease in $\delta^{15} \mathrm{~N}$ composition of soil with increasing distance from the hotspot resulting in a $0.06 \%$ decrease per $\mathrm{cm}$. (b) $\mathrm{C}: \mathrm{N}$ ratios exhibit an overall increase with increasing distance from the hotspot center, roughly 0.07 per $\mathrm{cm}$. 
Table S1: Soil biogeochemical data from carrion hotspots. Data from sampling dates 29 July 2016 to 9 December 2016 were previously published in Keenan et al. (2018).

\begin{tabular}{|c|c|c|c|c|c|c|c|c|c|c|}
\hline Sample & $\begin{array}{c}\text { Sampling } \\
\text { Date }\end{array}$ & Individual & $\begin{array}{c}\text { Decay } \\
\text { stage }\end{array}$ & $\begin{array}{c}\text { Dissolved } \\
\text { Oxygen } \\
(\%)\end{array}$ & $\delta^{15} \mathrm{~N}$ & $\delta^{13} \mathbf{C}$ & $\begin{array}{c}\text { Total } \\
\text { nitrogen } \\
(\%)\end{array}$ & $\begin{array}{c}\text { Total } \\
\text { carbon }(\%)\end{array}$ & $C: N$ & $\begin{array}{c}\text { Soil } \\
\text { Gravimetric } \\
\text { Moisture }\end{array}$ \\
\hline EAG1_R1 & 29-Jul-16 & 0 & Initial & 98.7 & 1.54 & -27.78 & 0.2998 & 5.132 & 17.118 & 0.301 \\
\hline EAG1_R2 & 29-Jul-16 & 0 & Initial & 98.7 & 1.24 & -27.89 & 0.301 & 5.2 & 17.276 & 0.286 \\
\hline EAG1_R3 & 29-Jul-16 & 0 & Initial & 98.7 & 1.68 & -27.93 & 0.284 & 5.001 & 17.609 & 0.311 \\
\hline EAG1_0108_B1 & 1-Aug-16 & 1 & Early & N.M. & 1.27 & -28.15 & 0.32 & 4.876 & 15.238 & 0.274 \\
\hline EAG1_0108_B2 & 1-Aug-16 & 2 & Early & N.M. & 4.96 & -27.34 & 0.164 & 2.323 & 14.165 & 0.186 \\
\hline EAG1_0108_B3 & 1-Aug-16 & 3 & Early & N.M. & 0.82 & -28.16 & 0.266 & 4.287 & 16.117 & 0.211 \\
\hline EAG1_0108_B4 & 1-Aug-16 & 4 & Early & N.M. & 3.14 & -27.66 & 0.255 & 4.779 & 18.741 & 0.206 \\
\hline EAG1_0108_B5 & 1-Aug-16 & 5 & Early & N.M. & 3.06 & -27.31 & 0.219 & 3.372 & 15.397 & 0.202 \\
\hline EAG1_0108_control & 1-Aug-16 & 0 & Control & N.M. & 1.76 & -27.56 & 0.247 & 4.196 & 16.988 & 0.219 \\
\hline EAG1_0308_B1 & 3-Aug-16 & 1 & Active & 20.7 & 6.16 & -27.94 & 0.4 & 4.755 & 11.888 & 0.276 \\
\hline EAG1_0308_B2 & 3-Aug-16 & 2 & Active & 1.6 & 7.22 & -27.68 & 0.233 & 2.834 & 12.163 & 0.193 \\
\hline EAG1_0308_B3 & 3-Aug-16 & 3 & Active & 2 & 5.14 & -28.01 & 0.447 & 4.83 & 10.807 & 0.295 \\
\hline EAG1_0308_B4 & 3-Aug-16 & 4 & Active & 9.7 & 9.16 & -27.38 & 0.303 & 4.167 & 13.752 & 0.245 \\
\hline EAG1_0308_B5 & 3-Aug-16 & 5 & Active & 11.8 & 5.67 & -27.36 & 0.254 & 3.512 & 13.827 & 0.161 \\
\hline EAG1_0308_control & 3-Aug-16 & 0 & Control & 98.6 & 1.48 & -27.68 & 0.267 & 4.159 & 15.577 & 0.160 \\
\hline EAG1_0908_B1 & 9-Aug-16 & 1 & Advanced & 1.8 & 7.24 & -27.74 & 0.251 & 3.356 & 13.371 & 0.269 \\
\hline EAG1_0908_B2 & 9-Aug-16 & 2 & Advanced & 16.7 & 7.12 & -27.89 & 0.316 & 4.101 & 12.978 & 0.297 \\
\hline EAG1_0908_B3 & 9-Aug-16 & 3 & Advanced & 1.4 & 8.53 & -27.54 & 0.237 & 2.952 & 12.456 & 0.240 \\
\hline EAG1_0908_B4 & 9-Aug-16 & 4 & Advanced & 3 & 12.26 & -27.65 & 0.4364 & 6.1946 & 14.195 & 0.418 \\
\hline EAG1_0908_B5 & 9-Aug-16 & 5 & Advanced & 74.1 & 8.44 & -27.35 & 0.275 & 3.401 & 12.367 & 0.205 \\
\hline EAG1_0908_control & 9-Aug-16 & 0 & Control & 98 & 1.26 & -27.77 & 0.281 & 5.008 & 17.822 & 0.223 \\
\hline
\end{tabular}


Table S1 (continued):

\begin{tabular}{|c|c|c|c|c|c|c|c|c|c|}
\hline Sample & $\begin{array}{c}\text { Soil } \\
\text { pH }\end{array}$ & Conductivity & $\begin{array}{c}\text { Nitrification } \\
\text { potential } \\
\text { rate (mg } \\
\mathrm{NO}_{2} \\
\text { /gdw/day) } \\
\end{array}$ & $\begin{array}{c}\text { Ammonium } \\
\left(\mathrm{mg} \mathrm{NH}_{4}-\mathrm{N}\right. \\
\left./ \mathrm{gdw}^{2}\right)\end{array}$ & $\begin{array}{c}\text { Microbial } \\
\text { respiration rate } \\
\left(\mu \mathrm{g} \mathrm{CO} \mathrm{CO}_{2}-\mathrm{C}\right. \\
\text { released/gdw/day })\end{array}$ & $\begin{array}{c}\text { DOC } \\
(\mathbf{m g} \\
\text { C/gdw) }\end{array}$ & $\begin{array}{l}\text { Protein } \\
(\mathrm{mg} / \mathrm{mL})\end{array}$ & $\begin{array}{c}\text { Nitrate } \\
\text { (mg NO }_{3}^{-} \\
-\mathrm{N} / \mathrm{gdw})\end{array}$ & $\begin{array}{c}\text { DON } \\
\text { (mg } \\
\text { N/gdw) }\end{array}$ \\
\hline EAG1_R1 & 6.71 & 51.49 & 0.214 & 0.040 & 48.721 & 2.325 & 0.309 & 0.000 & 0.270 \\
\hline EAG1_R2 & 6.82 & 41.01 & 0.204 & 0.032 & 44.542 & 2.341 & 0.219 & 0.000 & 0.276 \\
\hline EAG1_R3 & 6.85 & 51 & 0.124 & 0.045 & 59.392 & 2.663 & 0.224 & 0.000 & 0.305 \\
\hline EAG1_0108_B1 & 7.33 & 127.2 & 0.530 & 0.203 & 69.420 & 4.323 & 0.206 & 0.001 & 1.251 \\
\hline EAG1_0108_B2 & 6.94 & 67.99 & 0.187 & 0.068 & 29.033 & 2.600 & 0.141 & 0.000 & 0.588 \\
\hline EAG1_0108_B3 & 6.89 & 54.84 & 0.143 & 0.072 & 56.833 & 4.075 & 0.201 & 0.001 & 0.528 \\
\hline EAG1_0108_B4 & 6.59 & 73.87 & 0.161 & 0.118 & 65.631 & 4.044 & 0.244 & 0.000 & 0.853 \\
\hline EAG1_0108_B5 & 6.57 & 43.74 & 0.162 & 0.043 & 35.835 & 2.161 & 0.210 & 0.000 & 0.368 \\
\hline EAG1_0108_control & 6.82 & 36.78 & 0.167 & 0.011 & 35.200 & 2.346 & 0.159 & 0.000 & 0.242 \\
\hline EAG1_0308_B1 & 8.75 & 2757 & 0.425 & 3.152 & 270.062 & 92.596 & 0.241 & 0.000 & -0.126 \\
\hline EAG1_0308_B2 & 8.76 & 1352 & 0.343 & 1.647 & 296.125 & 40.543 & 0.265 & 0.000 & 1.200 \\
\hline EAG1_0308_B3 & 8.72 & 3737 & 0.393 & 3.650 & 417.638 & 115.727 & 0.270 & 0.000 & 0.816 \\
\hline EAG1_0308_B4 & 8.79 & 2474 & 0.442 & 3.261 & 342.022 & 69.771 & 0.295 & 0.000 & 0.086 \\
\hline EAG1_0308_B5 & 8.17 & 432.4 & 0.226 & 0.732 & 173.912 & 14.076 & 0.231 & 0.000 & 3.352 \\
\hline EAG1_0308_control & 6.68 & 31.65 & 0.163 & 0.010 & 27.807 & 2.516 & 0.225 & 0.000 & 0.205 \\
\hline EAG1_0908_B1 & 8.59 & 882.6 & 0.344 & 1.190 & 83.618 & 23.795 & 0.299 & 0.000 & 1.752 \\
\hline EAG1_0908_B2 & 8.88 & 1154 & 0.389 & 1.756 & 170.117 & 36.841 & 0.235 & -0.001 & 1.583 \\
\hline EAG1_0908_B3 & 8.89 & 1313 & 0.365 & 1.728 & 93.571 & 34.684 & 0.228 & 0.000 & 1.447 \\
\hline EAG1_0908_B4 & 8.85 & 2033 & 0.705 & 5.482 & 350.271 & 94.937 & 0.398 & 0.000 & 0.106 \\
\hline EAG1_0908_B5 & 8.7 & 787 & 0.285 & 1.302 & 116.209 & 22.208 & 0.243 & 0.000 & 4.643 \\
\hline EAG1_0908_control & 6.84 & 43.42 & 0.181 & 0.015 & 45.676 & 3.437 & 0.239 & 0.000 & 0.304 \\
\hline
\end{tabular}


Table S1 (continued):

\begin{tabular}{|c|c|c|c|c|c|c|c|c|c|c|c|}
\hline Sample & $\begin{array}{c}\text { Sampling } \\
\text { Date }\end{array}$ & Individual & Decay stage & $\begin{array}{l}\text { Dissolved } \\
\text { Oxygen } \\
(\%)\end{array}$ & $\delta^{15} \mathbf{N}$ & $\delta^{13} \mathrm{C}$ & $\begin{array}{c}\text { Total } \\
\text { nitrogen } \\
(\%)\end{array}$ & $\begin{array}{c}\text { Total } \\
\text { carbon }(\%)\end{array}$ & C:N & $\begin{array}{c}\text { Soil } \\
\text { Gravimetric } \\
\text { Moisture }\end{array}$ & Sample \\
\hline EAG1_0609_B1 & 6-Sep-16 & 1 & Early skeletal & 98.8 & 7.23 & -27.77 & 0.294 & 3.711 & 12.622 & 0.220 & EAG1_0609_B \\
\hline EAG1_0609_B2 & 6-Sep-16 & 2 & Early skeletal & 97.1 & 8.77 & -28.16 & 0.347 & 4.09 & 11.787 & 0.266 & EAG1_0609_B' \\
\hline EAG1_0609_B3 & 6-Sep-16 & 3 & Early skeletal & 96.6 & 9.49 & -27.68 & 0.241 & 2.772 & 11.502 & 0.196 & EAG1_0609_B. \\
\hline EAG1_0609_B4 & 6-Sep-16 & 4 & Early skeletal & 97.1 & 11.50 & -27.79 & 0.3515 & 4.6976 & 13.364 & 0.353 & EAG1_0609_B \\
\hline EAG1_0609_B5 & 6-Sep-16 & 5 & Early skeletal & 97.6 & 9.33 & -27.31 & 0.229 & 2.779 & 12.135 & 0.178 & EAG1_0609_B: \\
\hline EAG1_0609_control & 6-Sep-16 & 0 & Control & 98.3 & 1.78 & -27.64 & 0.259 & 4.023 & 15.533 & 0.121 & EAG1_0609_co \\
\hline EAG1_0912_B1 & 9-Dec-16 & 1 & Late skeletal & 100 & 8.38 & -27.71 & 0.2618 & 3.17 & 12.108 & 0.293 & EAG1_0912_B \\
\hline EAG1_0912_B2 & 9-Dec-16 & 2 & Late skeletal & 100 & 7.70 & -27.35 & 0.2215 & 2.7662 & 12.488 & 0.277 & EAG1_0912_B? \\
\hline EAG1_0912_B3 & 9-Dec-16 & 3 & Late skeletal & 100 & 8.97 & -27.63 & 0.1975 & 2.3564 & 11.931 & 0.277 & EAG1_0912_B: \\
\hline EAG1_0912_B4 & 9-Dec-16 & 4 & Late skeletal & 100 & 10.35 & -27.23 & 0.2011 & 2.7432 & 13.641 & 0.238 & EAG1_0912_B \\
\hline EAG1_0912_B5 & 9-Dec-16 & 5 & Late skeletal & 100 & 10.86 & -27.15 & 0.1866 & 2.3065 & 12.361 & 0.268 & EAG1_0912_B: \\
\hline EAG1_0912_control & 9-Dec-16 & 0 & Control & 100 & 1.79 & -27.30 & 0.1358 & 2.0874 & 15.371 & 0.246 & EAG1_0912_co \\
\hline EAG_1008_17_B1 & 8-Aug-17 & 1 & 1 yr post decay & N.M. & 6.64 & -27.89 & 0.336 & 4.608 & 13.730 & 0.425 & EAG_1008_17_ \\
\hline EAG_1008_17_B2 & 8-Aug-17 & 2 & $1 \mathrm{yr}$ post decay & N.M. & 7.28 & -27.48 & 0.33 & 4.08 & -6.73 & 0.367 & EAG_1008_17_ \\
\hline EAG_1008_17_B2 & 8-Aug-17 & 2 & 1 yr post decay & N.M. & 6.93 & -27.59 & 0.21 & 4.03 & -6.85 & 0.367 & EAG_1008 \\
\hline EAG_1008_17_B3 & 8-Aug-17 & 3 & 1 yr post decay & N.M. & 8.61 & -27.99 & 0.285 & 3.402 & 11.936 & 0.374 & EAG_1008_17_ \\
\hline EAG_1008_17_B4 & 8-Aug-17 & 4 & $1 \mathrm{yr}$ post decay & N.M. & 9.85 & -27.55 & 0.236 & 3.284 & 13.896 & 0.408 & EAG_1008_17_ \\
\hline EAG_1008_17_B5 & 8-Aug-17 & 5 & $1 \mathrm{yr}$ post decay & N.M. & 9.98 & -27.37 & 0.388 & 7.108 & 18.306 & 0.425 & EAG_1008_17_ \\
\hline EAG_1008_17_B5 & 8-Aug-17 & 5 & 1 yr post decay & N.M. & 9.83 & -27.43 & 0.199 & 4.727 & 23.772 & 0.425 & EAG_1008 \\
\hline EAG_1008_17_control & 8-Aug-17 & 0 & Control & N.M. & 0.05 & -27.74 & 0.255 & 4.358 & 17.083 & 0.462 & EAG_1008_17 \\
\hline
\end{tabular}


Table S1 (continued):

\begin{tabular}{|c|c|c|c|c|c|c|c|c|c|}
\hline Sample & $\begin{array}{l}\text { Soil } \\
\text { pH }\end{array}$ & Conductivity & $\begin{array}{l}\text { Nitrification } \\
\text { potential } \\
\text { rate }(\mathrm{mg} \\
\mathrm{NO}_{2} \\
\text { /gdw/day) } \\
\end{array}$ & $\begin{array}{c}\underset{\text { Ammonium }}{\left(\mathrm{mg} \mathrm{NH}_{4}-\mathrm{N}\right.} \\
\quad / \mathrm{gdw}) \\
\end{array}$ & $\begin{array}{c}\text { Microbial } \\
\text { respiration rate } \\
\left(\mu \mathrm{g} \mathrm{CO}_{2}-\mathrm{C}\right. \\
\text { released/gdw/day) }\end{array}$ & $\begin{array}{c}\text { DOC } \\
(\mathbf{m g} \\
\text { C/gdw) }\end{array}$ & $\begin{array}{l}\text { Protein } \\
(\mathrm{mg} / \mathrm{mL})\end{array}$ & $\begin{array}{l}\text { Nitrate } \\
\left(\mathrm{mg} \mathrm{NO}_{3}^{-}\right. \\
-\mathrm{N} / \mathrm{gdw}) \\
\end{array}$ & $\begin{array}{c}\text { DON } \\
(\mathbf{m g} \\
\text { N/gdw) } \\
\end{array}$ \\
\hline EAG1_0609_B1 & 7.25 & 693.8 & 10.859 & 0.545 & 52.499 & 9.960 & 0.188 & 0.191 & 4.776 \\
\hline EAG1_0609_B2 & 7.84 & 1107 & 0.974 & 0.874 & 146.121 & 12.483 & 0.235 & 0.567 & 7.451 \\
\hline EAG1_0609_B3 & 7.48 & 1146 & 9.005 & 0.739 & 45.885 & 14.282 & 0.205 & 0.328 & 6.053 \\
\hline EAG1_0609_B4 & 7.19 & 1121 & 11.875 & 0.913 & 87.189 & 18.769 & 0.314 & 0.333 & 7.217 \\
\hline EAG1_0609_B5 & 8.14 & 801.3 & 10.150 & 0.806 & 53.040 & 16.101 & 0.248 & 0.127 & 4.947 \\
\hline EAG1_0609_control & 6.84 & 35.08 & 0.130 & 0.006 & 20.816 & 2.889 & 0.193 & 0.000 & 0.185 \\
\hline EAG1_0912_B1 & 6.61 & 144.6 & -0.139 & 0.177 & 59.093 & 5.607 & 0.255 & 0.014 & 1.504 \\
\hline EAG1_0912_B2 & 7.13 & 122 & -0.228 & 0.209 & 42.560 & 5.134 & 0.246 & 0.013 & 1.556 \\
\hline EAG1_0912_B3 & 7.26 & 274.3 & 0.075 & 0.292 & 38.318 & 6.927 & 0.251 & 0.028 & 2.124 \\
\hline EAG1_0912_B4 & 6.55 & 301.3 & 0.143 & 0.221 & 101.803 & 24.986 & 0.268 & 0.021 & 2.294 \\
\hline EAG1_0912_B5 & 7.12 & 283.8 & 0.238 & 0.330 & 44.228 & 8.211 & 0.231 & 0.018 & 2.168 \\
\hline EAG1_0912_control & 6.73 & 29.13 & 0.039 & 0.012 & 46.453 & 3.121 & 0.195 & 0.001 & 0.309 \\
\hline EAG_1008_17_B1 & 6.46 & 23.98 & 0.005 & 0.008 & 65.516 & 3.424 & 0.205 & 0.000 & 0.094 \\
\hline $\begin{array}{l}\text { EAG_1008_17_B2 } \\
\text { EAG_1008_17_B2 }\end{array}$ & 6.23 & 21.20 & 0.006 & 0.006 & 56.631 & 2.957 & 0.240 & 0.000 & 0.087 \\
\hline EAG_1008_17_B3 & 5.86 & 28.47 & 0.006 & 0.006 & 56.187 & 2.007 & 0.242 & 0.000 & 0.082 \\
\hline EAG_1008_17_B4 & 5.74 & 40.00 & 0.006 & 0.007 & 68.760 & 3.151 & 0.261 & 0.001 & 0.120 \\
\hline $\begin{array}{l}\text { EAG_1008_17_B5 } \\
\text { EAG_1008_17_B5 }\end{array}$ & 6.21 & 33.70 & 0.005 & 0.014 & 73.221 & 2.529 & 0.246 & 0.001 & 0.091 \\
\hline EAG_1008_17_control & 6.29 & 23.07 & 0.006 & 0.007 & 59.451 & 2.429 & 0.195 & 0.000 & 0.093 \\
\hline
\end{tabular}


Table S2: Stable isotopic data for all vertical and lateral soil samples.

\begin{tabular}{|c|c|c|c|c|c|c|c|c|c|c|}
\hline Sample Name & $\begin{array}{c}\text { Date } \\
\text { Collected }\end{array}$ & $\begin{array}{c}\text { Beaver } \\
\text { number }\end{array}$ & $\begin{array}{l}\text { Depth } \\
\text { (cm) }\end{array}$ & $\begin{array}{c}\text { Distance } \\
\text { from } \\
\text { hotspot } \\
\text { (cm) }\end{array}$ & $\delta^{15} N$ & $\begin{array}{c}\text { Total } \\
\text { nitrogen } \\
(\%) \\
\end{array}$ & $\delta^{13} \mathrm{C}$ & $\begin{array}{c}\text { Total } \\
\text { carbon }(\%) \\
\end{array}$ & $\mathrm{C}: \mathrm{N}$ & $\begin{array}{c}\text { Gravimetric } \\
\text { moisture }\end{array}$ \\
\hline EAG_1008_D10_B1 & 8-Aug-17 & 1 & 10 & 0 & 6.50 & 0.11 & -26.39 & 1.54 & 14.49 & 0.250 \\
\hline EAG_1008_D10_B1 & 8-Aug-17 & 1 & 10 & 0 & 6.13 & 0.13 & -26.63 & 1.62 & 12.73 & 0.250 \\
\hline EAG_1008_D10_B2 & 8-Aug-17 & 2 & 10 & 0 & 5.74 & 0.14 & -26.97 & 1.95 & 14.14 & 0.265 \\
\hline EAG_1008_D10_B2 & 8-Aug-17 & 2 & 10 & 0 & 5.33 & 0.14 & -26.84 & 1.82 & 13.25 & 0.265 \\
\hline EAG_1008_D10_B3 & 8-Aug-17 & 3 & 10 & 0 & N.M. & N.M. & N.M. & N.M. & N.M. & 0.244 \\
\hline EAG_1008_D10_control & 8-Aug-17 & 0 & 10 & 0 & 3.29 & 0.11 & -26.54 & 1.78 & 16.77 & 0.332 \\
\hline EAG_1008_D15_B1 & 8-Aug-17 & 1 & 15 & 0 & 8.33 & 0.07 & -25.86 & 1.04 & 14.66 & 0.244 \\
\hline EAG_1008_D15_B2 & 8-Aug-17 & 2 & 15 & 0 & 6.57 & 0.11 & -26.13 & 1.23 & 11.66 & 0.213 \\
\hline EAG_1008_D15_B3 & 8-Aug-17 & 3 & 15 & 0 & 7.70 & 0.07 & -25.87 & 0.82 & 11.03 & 0.203 \\
\hline EAG_1008_D15_control & 8-Aug-17 & 0 & 15 & 0 & 6.61 & 0.07 & -25.29 & 0.88 & 13.09 & 0.263 \\
\hline EAG_1008_D20_B1 & 8-Aug-17 & 1 & 20 & 0 & 9.37 & 0.06 & -25.87 & 0.75 & 12.31 & 0.235 \\
\hline EAG_1008_D20_B2 & 8-Aug-17 & 2 & 20 & 0 & 7.50 & 0.08 & -25.87 & 1.05 & 13.82 & 0.205 \\
\hline EAG_1008_D20_B3 & 8-Aug-17 & 3 & 20 & 0 & 8.12 & 0.06 & -25.34 & 0.72 & 12.13 & 0.199 \\
\hline EAG_1008_D20_control & 8-Aug-17 & 0 & 20 & 0 & 7.76 & 0.05 & -24.52 & 0.55 & 10.21 & 0.332 \\
\hline EAG_1008_D30_B1 & 8-Aug-17 & 1 & 30 & 0 & 8.17 & 0.06 & -26.20 & 0.48 & 8.38 & 0.213 \\
\hline EAG_1008_D30_B2 & 8-Aug-17 & 2 & 30 & 0 & 8.05 & 0.07 & -25.75 & 0.92 & 12.36 & 0.234 \\
\hline EAG_1008_D30_B3 & 8-Aug-17 & 3 & 30 & 0 & 8.80 & 0.03 & -24.28 & 0.34 & 13.48 & 0.193 \\
\hline EAG_1008_D30_control & 8-Aug-17 & 0 & 30 & 0 & 6.14 & 0.05 & -25.44 & 0.60 & 11.34 & 0.232 \\
\hline EAG_1008_D40_B1 & 8-Aug-17 & 1 & 40 & 0 & 8.90 & 0.03 & -23.06 & 0.11 & 3.28 & 0.202 \\
\hline EAG_1008_D40_B1 & 8-Aug-17 & 1 & 40 & 0 & 8.64 & 0.03 & -23.30 & 0.17 & 5.13 & 0.202 \\
\hline$E A G \_1008 \_D 40 \_B 2$ & 8-Aug-17 & 2 & 40 & 0 & N.M. & N.M. & N.M. & N.M. & N.M. & 0.204 \\
\hline EAG_1008_D40_B3 & 8-Aug-17 & 3 & 40 & 0 & 8.56 & 0.03 & -24.67 & 0.24 & 8.43 & 0.184 \\
\hline EAG_1008_D40_control & 8-Aug-17 & 0 & 40 & 0 & 8.54 & 0.04 & -24.09 & 0.28 & 7.49 & 0.223 \\
\hline EAG_1008_D40_control & 8-Aug-17 & 0 & 40 & 0 & 8.36 & 0.04 & -23.41 & 0.29 & 7.56 & 0.223 \\
\hline
\end{tabular}


Table S2 (continued):

\begin{tabular}{|c|c|c|c|c|c|c|c|c|c|c|}
\hline Sample Name & $\begin{array}{c}\text { Date } \\
\text { Collected }\end{array}$ & $\begin{array}{c}\text { Beaver } \\
\text { number }\end{array}$ & $\begin{array}{c}\text { Depth } \\
\text { (cm) }\end{array}$ & $\begin{array}{c}\text { Distance } \\
\text { from } \\
\text { hotspot } \\
(\mathrm{cm})\end{array}$ & $\delta^{15} \mathbf{N}$ & $\begin{array}{c}\text { Total } \\
\text { nitrogen } \\
(\%) \\
\end{array}$ & $\delta^{13} \mathrm{C}$ & $\begin{array}{c}\text { Total } \\
\text { carbon } \\
(\%) \\
\end{array}$ & $\mathrm{C}: \mathbf{N}$ & $\begin{array}{c}\text { Gravimetric } \\
\text { moisture }\end{array}$ \\
\hline EAG_1008_D5_B1 & 8-Aug-17 & 1 & 5 & 0 & 5.38 & 0.18 & -27.09 & 2.61 & 14.09 & 0.332 \\
\hline EAG_1008_D5_B2 & 8-Aug-17 & 2 & 5 & 0 & 6.05 & 0.23 & -27.56 & 3.21 & 13.78 & 0.345 \\
\hline EAG_1008_D5_B2 & 8-Aug-17 & 2 & 5 & 0 & 5.91 & 0.18 & -27.61 & 3.50 & 19.34 & 0.345 \\
\hline EAG_1008_D5_B3 & 8-Aug-17 & 3 & 5 & 0 & 4.71 & 0.15 & -27.33 & 2.17 & 14.57 & 0.282 \\
\hline EAG_1008_17_B4 & 8-Aug-17 & 4 & 5 & 0 & 9.85 & 0.24 & -27.55 & 3.28 & 13.90 & 0.412 \\
\hline EAG_1008_17_B5 & 8-Aug-17 & 5 & 5 & 0 & 9.98 & 0.39 & -27.37 & 7.11 & 18.31 & 0.427 \\
\hline EAG_1008_17_B5 & 8-Aug-17 & 5 & 5 & 0 & 9.83 & 0.20 & -27.43 & 4.73 & 23.77 & 0.427 \\
\hline EAG_1008_D5_control & 8-Aug-17 & 0 & 5 & 0 & 2.70 & 0.13 & -26.72 & 2.11 & 16.63 & 0.378 \\
\hline EAG_1508_S100_B1 & 15-Aug-17 & 1 & 0 & 100 & 0.20 & 0.27 & -28.10 & 5.05 & 18.63 & 0.457 \\
\hline EAG_1508_S100_B2 & 15-Aug-17 & 2 & 0 & 100 & 0.05 & 0.22 & -27.61 & 4.93 & 22.59 & 0.391 \\
\hline EAG_1508_S100_B3 & 15-Aug-17 & 3 & 0 & 100 & -0.60 & 0.23 & -27.71 & 5.48 & 23.93 & 0.452 \\
\hline EAG_1508_S120_B1 & 15-Aug-17 & 1 & 0 & 120 & 0.00 & 0.26 & -27.93 & 5.54 & 21.69 & 0.434 \\
\hline EAG_1508_S120_B2 & 15-Aug-17 & 2 & 0 & 120 & -0.29 & 0.23 & -27.84 & 5.39 & 23.00 & 0.396 \\
\hline EAG_1508_S120_B3 & 15-Aug-17 & 3 & 0 & 120 & 0.04 & 0.21 & -27.05 & 5.97 & 28.81 & 0.440 \\
\hline EAG_1508_S140_B1 & 15-Aug-17 & 1 & 0 & 140 & 0.53 & 0.25 & -27.34 & 5.33 & 21.16 & 0.439 \\
\hline EAG_1508_S140_B2 & 15-Aug-17 & 2 & 0 & 140 & -0.26 & 0.23 & -27.76 & 4.55 & 19.66 & 0.430 \\
\hline EAG_1508_S140_B3 & 15-Aug-17 & 3 & 0 & 140 & -0.22 & 0.23 & -26.97 & 7.73 & 34.29 & 0.522 \\
\hline EAG_1508_S20_B1 & 15-Aug-17 & 1 & 0 & 20 & 5.30 & 0.31 & -27.76 & 4.50 & 14.56 & 0.393 \\
\hline EAG_1508_S20_B2 & 15-Aug-17 & 2 & 0 & 20 & 6.80 & 0.33 & -27.45 & 4.50 & 13.46 & 0.398 \\
\hline EAG_1508_S20_B2 & 15-Aug-17 & 2 & 0 & 20 & 6.15 & 0.23 & -27.58 & 4.14 & 18.14 & 0.398 \\
\hline EAG_1508_S20_B3 & 15-Aug-17 & 3 & 0 & 20 & 4.14 & 0.23 & -27.92 & 3.40 & 14.71 & 0.334 \\
\hline EAG_1508_S40_B1 & 15-Aug-17 & 1 & 0 & 40 & 2.73 & 0.31 & -27.69 & 5.32 & 17.26 & 0.438 \\
\hline EAG_1508_S40_B2 & 15-Aug-17 & 2 & 0 & 40 & 1.72 & 0.22 & -27.58 & 4.08 & 18.42 & 0.339 \\
\hline EAG_1508_S40_B3 & 15-Aug-17 & 3 & 0 & 40 & 2.27 & 0.19 & -27.52 & 2.67 & 13.86 & 0.287 \\
\hline EAG_1508_S40_B3 & 15-Aug-17 & 3 & 0 & 40 & 2.10 & 0.17 & -27.51 & 2.52 & 14.81 & 0.287 \\
\hline EAG_1508_S60_B1 & 15-Aug-17 & 1 & 0 & 60 & 0.71 & 0.28 & -27.61 & 5.26 & 18.68 & 0.444 \\
\hline EAG_1508_S60_B2 & 15-Aug-17 & 2 & 0 & 60 & 0.34 & 0.21 & -27.65 & 4.89 & 23.25 & 0.395 \\
\hline EAG_1508_S60_B3 & 15-Aug-17 & 3 & 0 & 60 & 0.82 & 0.21 & -27.92 & 3.30 & 15.70 & 0.330 \\
\hline EAG_1508_S80_B1 & 15-Aug-17 & 1 & 0 & 80 & 0.17 & 0.29 & -27.96 & 5.61 & 19.10 & 0.451 \\
\hline EAG_1508_S80_B2 & 15-Aug-17 & 2 & 0 & 80 & 0.04 & 0.22 & -27.85 & 4.91 & 22.62 & 0.420 \\
\hline EAG_1508_S80_B3 & 15-Aug-17 & 3 & 0 & 80 & -0.28 & 0.22 & -27.71 & 4.05 & 18.08 & 0.354 \\
\hline
\end{tabular}




\section{References}

Keenan, S.W., Schaeffer, S.M., Jin, V.L., DeBruyn, J.M., 2018. Mortality hotspots: nutrient cycling in forest soils during vertebrate decomposition. Soil Biology \& Biochemistry 121, 165-176. 